\title{
Pattern Definition of Foil Based Sensors with Ultrafast UV Lasers
}

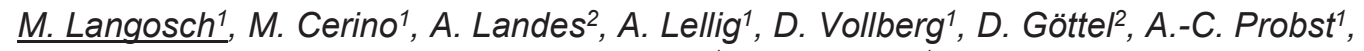 \\ O. Freitag-Weber ${ }^{1}$, G. Schultes ${ }^{1}$ \\ ${ }^{1}$ Hochschule für Technik und Wirtschaft des Saarlandes, 66117 Saarbrücken, Germany, \\ Matthaeus.Langosch@htwsaar.de \\ 2 Zentrum für Mechatronik und Automatisierungstechnik gemeinnützige GmbH, Gewerbepark \\ Eschberger Weg, 66121 Saarbrücken, Germany
}

\begin{abstract}
:
Our contribution demonstrates a laser-based patterning technique of a functional thin film on foils of polyimide. Using an ultrafast UV laser, it is possible to remove a highly strain sensitive layer that is based on nickel containing hydrocarbon compounds from the substrate and to create effortlessly an arbitrary design of foil strain gauges in the micrometer range. Furthermore the trimming performance of the laser on diverse designs are discussed. Ultrafast UV lasers are not only a powerful and flexible production tool for foil based sensors but facilitate also prototyping of new layouts for future applications.
\end{abstract}

Key words: laser based patterning technique, thin film, ultrafast UV laser, foil strain gauges, prototyping

\section{Background and Motivation}

The increasing demand for greater flexibility and lower production costs requires an appropriate fabrication process for foil strain gauges. However, the standard photolithography process chains have limitations. In particular, the inflexible and costly photo mask technique prevents an economical production of small quantities and is unsuitable for prototyping. Especially for high precision strain detection the requirements for the foil strain gauges are high and an adapted sensor layout is needed [1].

On the other hand, it is known that pulsed UV lasers eliminate the need of photo masks and allow a direct material patterning in specific cases $[2,3]$. For example, highly strain sensitive thin film structures based on nickel containing hydrocarbon compounds (Ni:a-C:H, nanoNi@C) for pressure sensors are structured without damaging the underlying insulating layer of $\mathrm{SiO}_{2}[4]$.

The aim of this work is to show the possibilities of an ultrafast UV laser in the production of thin film strain gauges on polyimide $(\mathrm{Pl})$. Starting with the influence of the laser on the coated $\mathrm{PI}$ foil, demonstrating direct writing, also the resistor trimming will be discussed.

\section{Method}

The starting point is a $50 \mu \mathrm{m}$ thick $\mathrm{PI}$ foil covered with a piezoresistive thin layer with a thickness of approx. $200 \mathrm{~nm}$. For this purpose, highly strain sensitive thin films based on Ni:a$\mathrm{C}: \mathrm{H}$ were deposited onto polyimide by means of reactive sputtering processes $[5,6,7]$. An ultrafast $\mathrm{Nd}: \mathrm{YVO}_{4}$ laser with the higher harmonic wavelength at $355 \mathrm{~nm}$ and a pulse duration of $<15$ ps serves to ablate parts of this layer locally. The mark speed can be selected up to $800 \mathrm{~mm} / \mathrm{s}$. The laser spot diameter of about $15 \mu \mathrm{m}$ is theoretically the only restriction in designing the structure and performing the resistor trimming.

An ex situ trimming is performed to adjust the resistance of the laser structured foil strain gauges. After structuring of the pattern definition values of electrical resistance are measured in an additional setup. Afterwards the specimens are processed again by laser to achieve a certain resistance value. The here demonstrated ex situ trim algorithm is as follows. After choosing of a trim geometry the influence on the resistance has to be investigated. For this purpose the foil strain gauges are trimmed with varying trim geometry sizes to obtain a trim function. Depending on the geometry a linear behavior of the relative resistance change to the size of the trim 
geometry is not always valid. For a certain relative resistance change to a higher value the necessary size has to be interpolated.

\section{Results and Discussion}

The influence of the laser on the coated PI foil is shown in figure 1. The three lines on the coated surface are created with the scan velocities 50,225 and $400 \mathrm{~mm} / \mathrm{s}$ and at the sequence repetition rate of $160 \mathrm{kHz}$. To avoid a carbonization of the $\mathrm{PI}$ foil the scan velocity has to be increased. In general it can also be realized with smaller repetition rates. Furthermore the functional layer near the carbonized PI foil is significantly influenced and shows a wave like structure. Increasing the velocity in respect to the repetition rate, parameters can be found that allow the ablation of the functional layer with only a small destruction of the PI foil. The foil shows no carbonization and an electrically insulation is achieved.

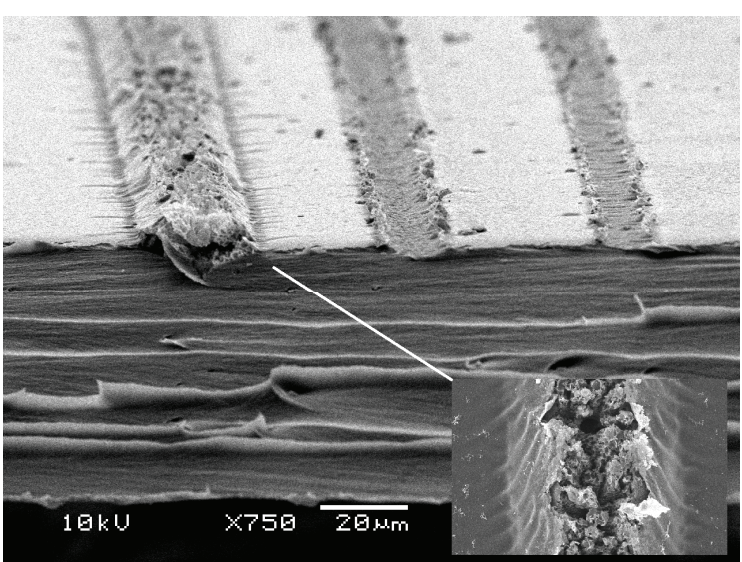

Fig. 1. SEM images of a line structured PI foil that is coated with Ni:a-C:H using three different scan velocities. The left line shows carbonized PI foil, the right one an ablation of the $200 \mathrm{~nm}$ thick functional layer without any significant ablation of the PI foil.

The detailed influence of the laser is shown in figure 2 . The line was written at a scan velocity of $400 \mathrm{~mm} / \mathrm{s}$ and the repetition rate of $80 \mathrm{kHz}$. In the middle the ablation of a small amount of $\mathrm{PI}$ foil is visible. Additionally the overlap of the pulses can be recognized. The transition to the untouched functional layer shows percolated nickel and flakes of the residual layer.

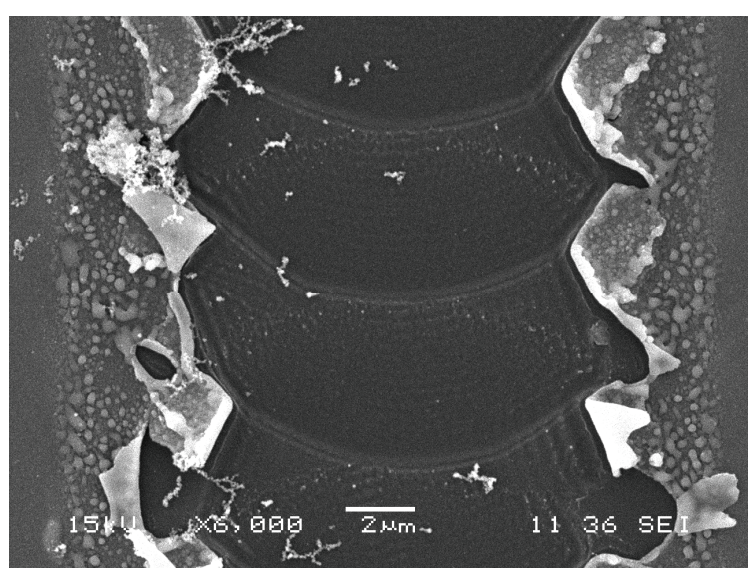

Fig. 2. SEM image of a structured coated PI foil.

However the detailed laser parameters depend on the material composition and thickness. Furthermore intensities can be chosen that ablate significantly the $\mathrm{PI}$ foil, but create a sharper border as shown in the figures 1 and 2 . At least the path width of the foil strain gauge is an important factor in the choice of the laser parameters. For wider paths border errors become less significant.
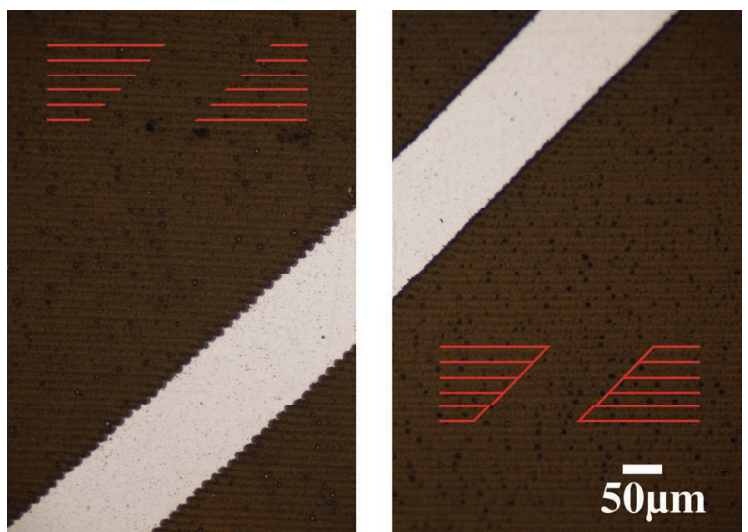

Fig. 3. Images of structured foil strain gauges. Bright: strain sensitive layer. Dark: polyimide after removal of the strain sensitive layer. Red: illustration of the laser scan path. Left: removal by means of hatching. Right: removal with additional contour cut. The path width is $120 \mu \mathrm{m}$.

A large-scale material removal is performed via a hatch with a line distance smaller than the laser spot. An example of the removal of the functional layer with additional ablation of $\mathrm{PI}$ is shown in figure 3 . The dark places indicate polyimide. The bright one is the strain sensitive thin film. To obtain smooth borders a contour cut is required. In this case, by ablating the upper layer a removal of polyimide of the thickness of about $1 \mu \mathrm{m}$ occurs as well. It has to be mentioned that there is no carbonization of the polyimide. Accordingly, an insulation can be ensured. A further benefit of the possibility to remove a certain amount of the substrate is to control the strain transfer to the gauge pattern. 

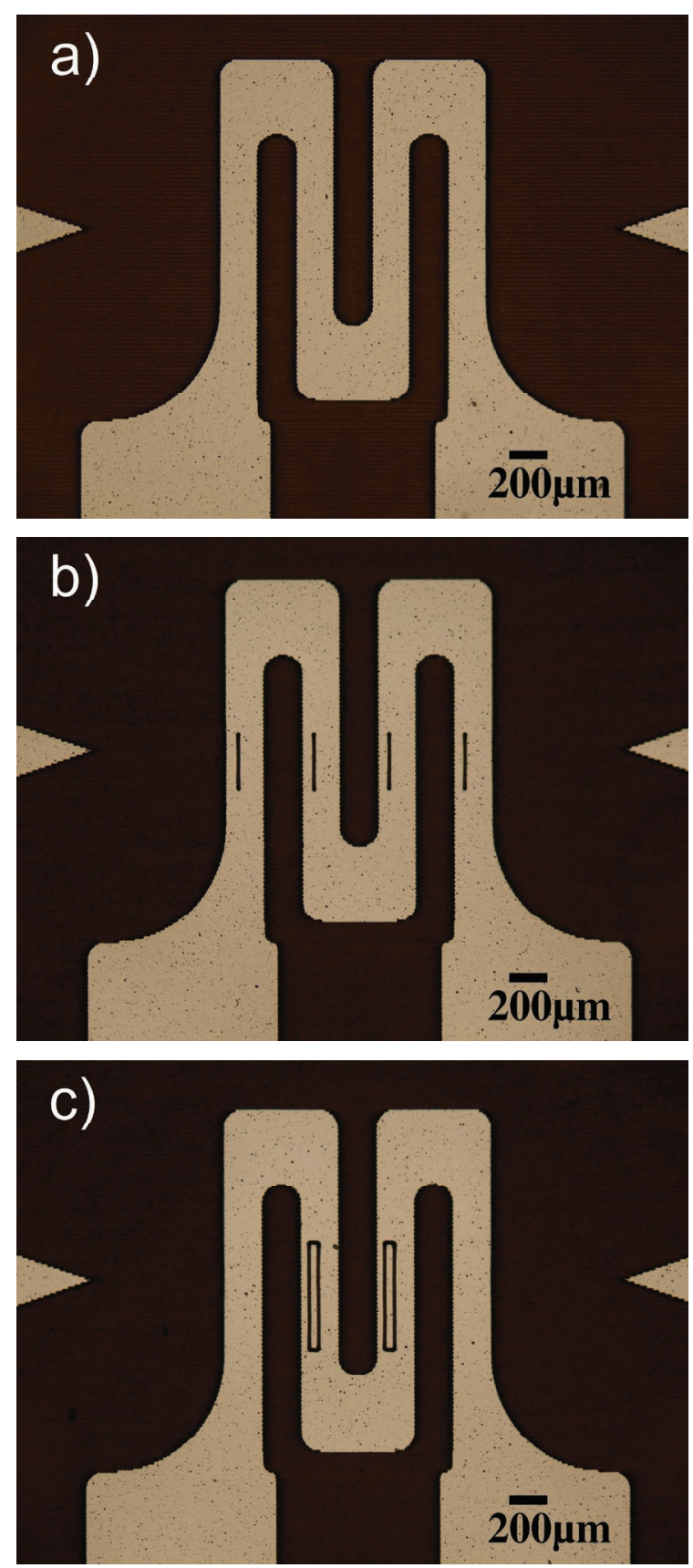

Fig. 4. Images of laser trimmed foil strain gauges. (a): initial situation. (b): trimming by lines. (c) trimming by squares.

In general, the structured foil strain gauges have to be trimmed to a desired resistance value. For this purpose, the ultrafast UV laser is suitable, too. Different correction geometries are suitable to obtain the desired resistance value. An example of two different geometries is shown in Fig. 4. The length of the lines (b) or the area of the squares (c) depends on the deviation from the desired value. Two points are important in the election of the right geometry. The first is the size of the correction resulting from the difference to the specified value. Higher changes of the resistance requires extensive interventions in the layout. For small changes a small cut as shown in figure 4 (b) is sufficient. On the other hand, in the case of ex situ trimming a geometry is preferable which requires less accuracy in positioning after the resistance measurement.
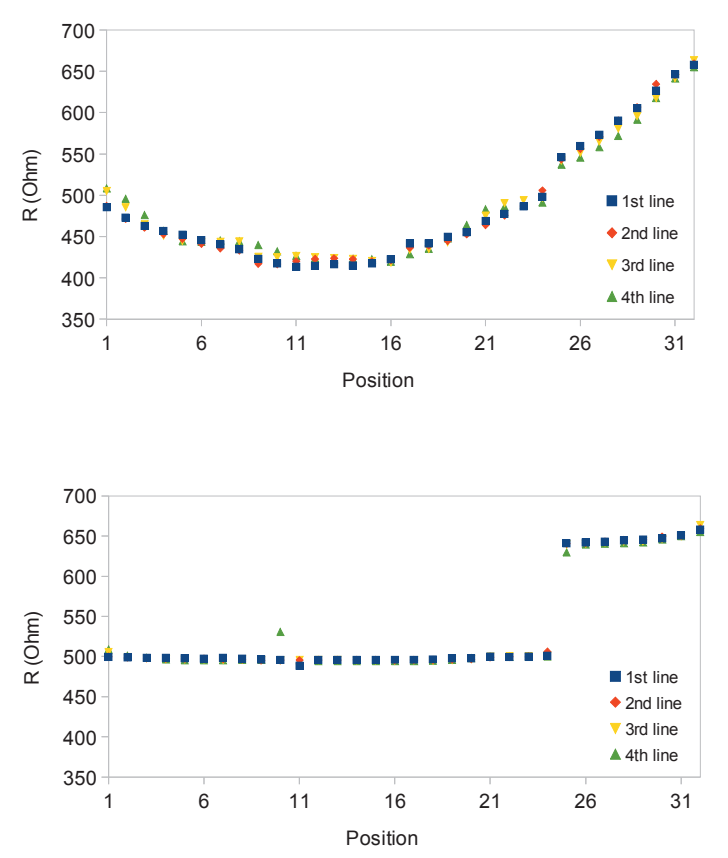

Fig. 5. Top: resistance values of 128 foil strain gauges dependent on their position in a sputter device. The lines indicate the machine direction. Bottom: the resistance values after trimming.

A result of the trim algorithm is shown in figure 5 . In this case 128 gauges were structured along four lines. The lines show the machine direction. The inhomogeneity of the reactive sputtering process leads to a variation of the resistance values of the foil strain gauges across the coated $\mathrm{PI}$ foil in transvers direction. The application of the trim algorithm on each strain foil gauges allows a resistance increase to $500 \Omega$ (position 1 to 24). The residual structures were trimmed to a resistance value of $650 \Omega$.

In general, the performance of the trimming is dependent on several factors. The quality of the trim function has to be pointed out. Impurities in the thin layer can lead to errors which are reflected in the trimmed gauges. In this case it is of advantage to smooth the curve of the trim function. Inconvenient are strong local inhomogeneities due to the assumption that the resistivity within a structure is homogeneous. Furthermore the writing accuracy of about $1 \mu \mathrm{m}$ and the reproducibility of the sample positioning in the ex situ trim algorithm have to be taken into account. Nevertheless accuracies of less than $0.1 \%$ were measured. 


\section{Summary}

Ultrafast UV lasers are powerful tools for the pattern definition of foil based sensors. Laser parameters can be found depending on the thin layer and PI foil to ablate the functional layer with minimal destruction of the substrate. Furthermore an ablation of the layer with $\mathrm{PI}$ can be realized without carbonization of the PI foil, too. The electrical insulation can be ensured in both cases. This allows a structuring and trimming of thin film foil gauges. For example, an ex situ trim algorithm was applied, which has provided resistance accuracies less than $0.1 \%$.

\section{References}

[1] H. Grange, C. Maeder, C. Bieth, S. Renard, G. Delapierre, Sensors and Actuators A: Physical 46, 213-217 (1995); doi: 10.1016/09244247(94)00892-L

[2] K.-T. Kim, J.-Y. Oh, B.-S. Shin, D.-S. Park, Bulletin of the Korean Chemical Society 33, 3937-3938 (2012); doi: 10.5012/bkcs.2012.33.12.3937

[3] J.F. Duesing, O. Suttmann, J. Koch, U. Stute, L. Overmeyer, JLMN-Journal of Laser Micro/Nanoengineering 7, 3-311 (2012)

[4] M.Cerino, D. Goettel, G. Schultes, patent DE 102013011157.9 (2013)

[5] G. Schultes, P. Frey, D. Goettel, O. FreitagWeber, Diam. Relat. Mater. 15 (1), 80-89 (2006); doi:10.1016/j.diamond.2005.07.005

[6] R. Koppert, H. Schmid-Engel, S. Uhlig, A.-C. Probst, D. Goettel, G. Schultes, U. Werner, Diamond \& Related Materials 25, 50-58 (2012); doi: 10.1016/j.diamond.2012.01.031

[7] S. Uhlig,R. Struis, H. Schmid-Engel,J. Bock, A.C. Probst, O. Freitag-Weber, I. Zizak,R. Chernikov, G. Schultes, Diamond and Related Materials 34, 25-35 (2013)
The flexibility of the laser facilitates not only the convenient creation of an arbitrary design of foil strain gauges in the micrometer range, but also the controlled ablation of the substrate to control the strain transfer.

\section{Acknowledgment}

The present project is sponsored by the Federal Ministry of Education and Research, Germany (VIP "Hochempfindliche Folien-DMS", support code 03V0436). 